in which tolerance is acquired is obscure. With some of them it is difficult to find two patients who react to the same dose in exactly the same way; this holds not only for the antihistamine drugs proper but also, to a certain extent, for other substances with partly antihistamine action, like ephedrine and adrenaline. To mention only two of the many unexplained facts-why does anthisan, when given intravenously, require as long as 5 to 15 minutes until it acts? And how are we to explain that if ephedrine, "benadryl," or anthisan are given in one dose daily some of the side symptoms disappear after one or two days while other effects stay almost indefinitely?

Obviously these are very complex problems, and the question of the efficacy of these drugs cannot be solved by the use of dummy tablets and the reports of the patients. For this reason I feel that the conclusions in your leading article are premature.-I am, etc.

London, W.C.1.

H. HeRXHeimer.

\section{Penicillin Treatment of Scarlet Fever}

SIR,-An outbreak of this disease in a large institution afforded an opportunity on a small scale of trying the method oi treatment advocated by Dr. Torben Jersild, of the University of Copenhagen (Feb. 14, p. 318). Before seeing this report, 11 of the 79 cases had been treated with sulphanilamide with unsatisfactory results, inasmuch as three had unfavourable features. One had double antrum inflammation, another cervical adenitis, and the third went home after four weeks still harbouring the haemolytic streptococcus $\mathbf{A}$ in nose and throat and continuing to show positive swabs until nearly eight weeks from the commencement of the illness. Penicillin intramuscularly, as a snuff, and as pastilles caused a rapid disappearance of all symptoms and of the haemolytic streptococcus in the other two cases.

The remaining 68 had penicillin as recommended by Dr. Torben Jersild-100,000-150,000 units intramuscularly, twice a day, for six days. Not a single complication of any kind occurred, except in one case a penicillin rash was seen on the third day and treatment was stopped. It also had no effect in preventing recurrence of scarlet fever, for two cases had the typical rash and slight sore throat 3-4 weeks after the first attack. Penicillin treatment for three days resulted in negative swabs on the eighth day. Sixty-two gave negative nose and throat swabs when tested on or about the eighth day after the rash. Positive swabs were obtained in the six other cases; five became negative after about 14 days with further penicillin treatment for three days. One failed to respond until 24 days later, when very few haemolytic streptococci were found. A prolonged and intractable acute tonsillitis a few months previously may have been responsible for this poor result.

Most of these might safely have returned to the institution after eight days; but as it was winter a period of two weeks' isolation was adopted, and a further week for convalescence. The advantage of a shortening of the time lost through illness appears to be counterbalanced only by the objection that patients so treated may show penicillin sensitization at some future date. Rapid relief of sore throat was noticeable in all cases, and the general health excellent.

I am greatly indebted to Dr. D. D. Payne, the Medical Officer of Health, and Dr. J. Colbeck, County Pathologist, for their kind and helpful co-operation.-I am, etc.,

Harrogate.

\section{R. F. Campbell Ward.}

\section{Diagnosis of Periarteritis Nodosa}

SIR,-The two cases of periarteritis nodosa described by Dr. J. M. Sutherland (May 1, p. 832) again bring out the difficulties of diagnosis. As Dr. Sutherland states in the section on diagnosis, "The possibility of periarteritis nodosa should be borne in mind in a case presenting multiple symptoms referable to several systems." Having arrived at this stage in the differential diagnosis, the problem arises as to how to clinch the diagnosis. The means of doing so fall into two groups, $(a)$ histological section of a node or piece of muscle, and $(b)$ visual evidence.

As regards the latter, Dr. Sutherland mentions only the ophthalmoscope, but there is another way, and that is visualization of the lesions through the peritoneoscope. Involvement of the mesenteric vessels is common in this condition, and it is easy to visualize the nodes on these vessels through a peritoneoscope. The procedure can be carried out under local anaesthesia with a minimum of disturbance to the patient. Dr. Sutherland's first patient had abdominal pain, and the necropsy in Case 2 revealed involvement of the vessels of the small intestine. It is almost certain that Case 1 would have shown mesenteric involvement. I have described the visualization of the condition by peritoneoscopy in an article in the Medical Press $(1947,118,487)$. I am, etc.,

Leicester.

A. P. M. PAGe.

\section{The Problem of Caries}

SIR, - I have recently had the opportunity to read the annotation (April 10, p. 697) on "The Problem of Caries." Since you call attention to the researches of Pincus and Frisbie, which supposedly support the concept that the destruction of the enamel organic matrix is the initial caries lesion, it seems opportune to point out some of the recent evidence supporting the more widely held view that surface enamel acid production is directly related to the caries process.

In our laboratories we have made the observation that following the ingestion of refined carbohydrates the glucose (reducing substance) content of the saliva may show an immediate elevation to values in excess of $1,000 \mathrm{mg} . \%$. This magnitude, depending upon the eating habits, characteristics of the food, etc., may persist in certain instances for periods as great as one hour. Stephan has demonstrated by in vivo methods that within two minutes after sugar solutions are ingested by caries-susceptible persons the $p \mathrm{H}$ of the tooth surface or within the carious cavity falls from approximately 7.0 to below 5.0. Such phenomena do not occur in cariesimmune subjects.

The significance of $p \mathbf{H}$ values approximating 4.5 is not clearly understood. A variety of possibilities present themselves. They include: (1) This $p \mathrm{H}$ is critical for enamel decalcification. (2) This $p \mathrm{H}$ approaches the optimum for the activity of the acid phosphatase, an enzyme which has been demonstrated to be present in salivary bacteria by at least three investigators. (3) This $p \mathrm{H}$ approaches the optimum for many proteolytic enzyme systems, possibly for those present in organisms capable of producing caries. (4) This $p \mathrm{H}$ approximates the iso-electric point of the enamel protein, and the physical properties of this tissue may undergo marked alterations of importance in caries production. Any or all of these may be related to caries aetiology, but each depends on refined carbohydrate intake and bacterial acid production.

One fact seems indisputable-the diet must contain refined carbohydrates of the nature of starch, sucrose, or glucose if we are to produce experimental caries in animals. The Syrian hamster has been studied extensively by American investigators since 1942. I am aware of no single instance in which experimental caries has been reported as being produced with diets devoid of these materials. Fortification of these experimental diets with so-called protective factors has been a most ineffective caries-control measure.

It also seems pertinent to mention that the most extensive caries-control measure being used in the United States to-day is the topical application of fluorides. These have been shown repeatedly by controlled clinical studies on human being and animals to reduce dental caries by slightly in excess of $40 \%$ Laboratory researches indicate that the application of topical fluoride results in a union of fluoride with the mineral phase of the enamel, reducing its solubility by approximately $50 \%$ and enhancing its surface hardness. It seems more than coincidental that a substance affecting acid solubility should limit dental caries.

One cannot resist the temptation of suggesting that the most unsatisfactory aspect to research in the field of dental caries has been the constant unscientific criticism of our endeavours by many medical colleagues. In general, these gentlemen refuse to accept the view that the aetiology of tooth decay may not be comparable to that of other diseases. Such an attitude is discouraging, but understandable when one remembers the reluctance with which such fundamental disease concepts as bacterial infection, virus infection, and vitamin deficiencies were adopted.-I am, etc.,

Tufts College Dental School, Boston, Mass.

\section{J. F. Volker.}

\section{The Process of Ageing}

SIR,-No doubt treatment of ovariectomized rats by various sex hormones and thyroid, as described by Dr. V. Korenchevsky (April 17, p. 728), represents valuable research work on relations and interactions of different endocrine glands and on their consequences to the metabolism of other organs, but it must be emphasized that ovariectomy itself does not produce the same effects as the physiological process of growing old in spite of certain features in common-for instance, in both cases 